\title{
A comparative study of emergence agitation between sevoflurane and propofol anesthesia in adults after closed reduction of nasal bone fracture
}

\author{
Young-Shin Kim, Young Keun Chae, Young Soon Choi, Jin-Hye Min, So Woon Ahn, Jong Won Yoon, \\ Sang Eun Lee, and Yong Kyung Lee
}

Department of Anesthesiology and Pain Medicine, Myongji Hospital, Kwandong University Medical School, Goyang, Korea

Background: Emergence agitation is associated with increased morbidity and hospital costs. However, there have been few reports in the medical literature on the occurrence of emergence agitation in adults. The aim of this study was to compare emergence agitation between sevoflurane and propofol anesthesia in adults after closed reduction of nasal bone fracture.

Methods: Forty adults (ASA I-II, 20-60 yr) undergoing closed reduction of nasal bone fracture were randomly assigned to either sevoflurane or propofol group and anesthesia was maintained with sevoflurane or propofol. The bispectral index (BIS) was monitored and maintained within 40-60. At the end of surgery, patients were transported to the post anesthetic care unit (PACU) and agitation state scale was checked by Aono's four-point scale (AFPS). Emergence agitation was defined as and AFPS score of 3 or 4 . Pain score were measured by numeric rating scale (NRS) on arrival and peak value at PACU.

Results: Nine (45.0\%) patients in the sevoflurane group and $2(10.0 \%)$ patients in the propofol group developed emergence agitation in the PACU $(\mathrm{P}=0.031)$. There was no correlation between peak NRS and Aono's four-point scale.

Conclusions: Propofol may decrease incidence of emergence agitation compared to sevoflurane in adults undergoing closed reduction of nasal bone fracture. (Korean J Anesthesiol 2012; 63: 48-53)

Key Words: Adult, Emergence agitation, Propofol, Sevoflurane.

\footnotetext{
Received: February 7, 2012. Revised: February 20, 2012. Accepted: February 22, 2011.

Corresponding author: Yong Kyung Lee, M.D., Department of Anesthesiology and Pain Medicine, Myongji Hospital, Kwandong University Medical School, 697-4, Hwajung-dong, Deokyang-gu, Goyang 412-270, Korea. Tel: 82-31-810-6325, Fax: 82-31-810-6209, E-mail: mdtweety@naver.com (c) This is an open-access article distributed under the terms of the Creative Commons Attribution Non-Commercial License (http:// creativecommons.org/licenses/by-nc/3.0/), which permits unrestricted non-commercial use, distribution, and reproduction in any medium, provided the original work is properly cited.
} 


\section{Introduction}

Closed reduction of nasal bone fracture is performed under general anesthesia due to the intense pain it causes, despite it being a short surgery. Many patients complain of discomfort in breathing because of the intranasal packing performed to prevent bleeding. Due to the short surgery time, and to prevent absorption of intranasal bleeding, it is preferable to use an anesthetic with rapid emergence. Sevoflurane has the characteristics of rapid induction and emergence of general anesthesia due to its low blood-gas partition coefficient and blood-tissue partition coefficient [1], hence it is an appropriate drug for general anesthesia in short surgeries such as closed reduction of nasal bone fracture. However, emergence agitation frequently occurs with sevoflurane; in particular pediatric patients, are known to have a higher incidence of emergence agitation with sevoflurane compared to other inhalation anesthetics $[2,3]$. There are reports that propofol has rapid anesthesia induction and emergence of general anesthesia. Moreover, compared to sevoflurane, it causes lower incidence of emergence agitation when anesthesia is maintained through intravenous administration [4].

Emergence agitation can often occur after anesthesia and can increase the risk of falling, bleeding, self-extubation, and removal of endotracheal tubes, and there is the need for continuous monitoring of patients by recovery room staffs and treatments such as drug administration or physical restraint of the patient [5]. However, most research regarding emergence agitation is conducted on pediatric patients, and there is little data on adult patients. Therefore, the authors in this study compared the incidence and degree of emergence agitation with sevoflurane and propofol, which are commonly used anesthetics, in adult patients scheduled for closed reduction of nasal bone fracture.

\section{Materials and Methods}

This study was approved by the Institutional Review Board of the hospital, and the purpose and method were explained to the patient and guardian prior to surgery to obtain informed consent. The study was conducted on 40 adult patients aged 2060 in American Society of Anesthesiologists class 1 or 2 who were scheduled for closed reduction of nasal bone fracture. Patients with signs or symptoms of infection in the upper respiratory tract, history of sleep apnea, administered medication for psychiatric diseases were excluded from the study. Using the table of random sampling numbers, the patients were randomly assigned to sevoflurane (group S) or propofol (group P). A medical attendant who was unaware of the grouping of the patients evaluated each patient's response in recovering from anesthesia during his or her stay in the recovery room.

All patients were transported to the operating room without preoperative medication, and the patients were reminded that there could be discomfort from nasal packing following the surgery. All patients had the following monitoring devices attached: electrocardiography, noninvasive blood pressure, pulse oximetry monitor, and BIS. While patients were being administered $100 \%$ oxygen through a face mask, they received fentanyl $1 \mu \mathrm{g} / \mathrm{kg}$ by IV injection. For the purposes of group comparison, patients in group $\mathrm{S}$ received thiopental $5 \mathrm{mg} / \mathrm{kg}$ by IV injection and group P received propofol $2 \mathrm{mg} / \mathrm{kg}$ by IV injection. Both groups of patients received $1 \mathrm{mg} / \mathrm{kg}$ of succinylcholine by IV injection. After fasciculation had passed, manual ventilation was continued, and anesthesia was maintained through sevoflurane 4 vol\% or by continuous injection of propofol at $200 \mu \mathrm{g} / \mathrm{kg} / \mathrm{min}$, according to group until BIS level reached 60 . After BIS reached 60, endotracheal intubation was performed, and anesthesia was then maintained through controlling the concentration of sevoflurane and the injection speed of propofol so the BIS value remained 40-60. Respiration rate and tidal volume were controlled so end-tidal $\mathrm{CO}_{2}$ concentration was maintained at $30-34 \mathrm{mmHg}$, and a total of $2 \mathrm{~L} / \mathrm{min}$ of nitrous oxide and oxygen was administered in a $1: 1$ ratio as inhalation gas. Additional opioids were not used during surgery in any of the patients, and when blood pressure showed a difference of more than $\pm 20 \%$ from basal levels, labetalol or ephedrine was injected IV for adjustment. All patients received bilateral nasal packs. After the completion of surgery when the operating surgeon attached the nasal splint, all anesthesia was ceased and $100 \%$ oxygen was administered to all patients. An anesthesiologist was assigned to observe the recovery state of each patient, and was responsible for the recovery. When spontaneous respiration and muscle strength of the patient recovered sufficiently to allow the patient to respond to verbal stimulation and after the patient's open eyes had opened, the endotracheal tube was removed and the patient was transported to the recovery room.

After the patient had opened his or her eyes as a response to language stimulation, the degree of emergence agitation of the patient was measured using Aono's four-point scale (Table 1). The degree of pain was investigated through a numeric rating scale (NRS) ( $0=$ no pain, $10=$ unimaginably severe pain). The measurements were repeated every 2 minutes to obtain the

Table 1. Aono's Four-Point Scale

\begin{tabular}{ll}
\hline Calm & 1 \\
Not calm, but could be easily calmed & 2 \\
Moderately agitated or restless & 3 \\
Combative, excited, disoriented & 4 \\
\hline
\end{tabular}


Table 2. Demographic and Perioperative Data

\begin{tabular}{lcc}
\hline & Group S (n=20) & Group P (n=20) \\
\hline Age (yr) & $33.00 \pm 2.73$ & $35.20 \pm 2.43$ \\
Sex $(\mathrm{M} / \mathrm{F})$ & $16 / 4$ & $15 / 5$ \\
Height $(\mathrm{cm})$ & $169.10 \pm 1.84$ & $168.45 \pm 1.43$ \\
Weight $(\mathrm{kg})$ & $66.41 \pm 1.74$ & $69.30 \pm 2.28$ \\
Duration of surgery (min) & $12.85 \pm 1.57$ & $14.85 \pm 1.19$ \\
Duration of anesthetic exposure (min) & $23.85 \pm 1.64$ & $22.25 \pm 1.31$ \\
Time from end of surgery to extubation (min) & $10.35 \pm 1.01$ & $10.95 \pm 0.89$ \\
Time to discharge from PACU (min) & $32.00 \pm 2.22$ & $33.30 \pm 1.58$ \\
Duration of agitation (min) & $0.98 \pm 0.25$ & $0.85 \pm 0.36$ \\
Peak NRS & $4.10 \pm 0.45$ & $4.90 \pm 0.41$ \\
Total amount of fentanyl in the PACU $(\mu \mathrm{g})$ & $25.00 \pm 6.54$ & $33.75 \pm 6.90$ \\
\hline
\end{tabular}

Values are mean \pm SD. Group S: sevoflurane group, Group P: propofol group, PACU: postanesthetic care unit, NRS: numeric rating scale, 0: no pain to 10: worst imaginable pain.

peak NRS and peak Aono's four-point scale scores. In addition, the exposure time to anesthetics, surgery time, time from end of surgery to extubation, and duration of emergence agitation were investigated. Patients received $25 \mu \mathrm{g}$ fentanyl IV when their NRS score was found to be higher than 3; the total amount of fentanyl administered was recorded. When emergence agitation continued for more than 3 minutes, $10 \mathrm{mg}$ of nalbuphine was administered IV. When emergence agitation continued for more than $1 \mathrm{~min}$ after drug administration, patients received $10 \mathrm{mg}$ nalbuphine was injected IV, repeatedly.

The patients were observed for the occurrence of complications or side effects, while being observed for nausea, vomiting, tremors, and hypersalivation. If a patient was experienced nausea or vomiting, the patient received $0.3 \mathrm{mg}$ ramosetron by IV; pethidine $25 \mathrm{mg}$ was administered IV to patients if they were experiencing tremors. When respiratory failure or laryngospasm occurred, assisted respiration was performed, and endotracheal intubation was performed again if considered necessary. Emergence agitation was defined to be when the Aono's four-point scale score was 3 or higher [6]. When the patient was hemodynamically stable, could maintain their own airway, and oxygen saturation was maintained at greater than $95 \%$ during atmospheric respiration, the patient was transported to the ward, and the duration of stay in the recovery room was recorded.

Data was expressed as mean \pm standard deviation, and SPSS version 12.0 was used in the statistical analysis. The t-test was used in the analysis of parametric data between the 2 groups, and the Mann-Whitney U test was used for non-parametric data. In the case of categorical data, the chi-square test was used, and the Fisher's exact test was used when necessary. Spearman's correlation was used to analyze the correlation between patient's pain and emergence agitation. Findings were considered to be statistically significant when the P value was less than 0.05 .
Table 3. Postoperative Aono's Four-Point Scale, Agitation

\begin{tabular}{lcc}
\hline & Group S $(\mathrm{n}=20)$ & Group P (n=20) \\
\hline Aono's four point scale & & \\
1 & $1(5.0 \%)$ & $14(70.0 \%)$ \\
2 & $10(50.0 \%)$ & $4(20.0 \%)$ \\
3 & $7(35 \%)$ & $2(10.0 \%)$ \\
4 & $2(10.0 \%)$ & $0(0.0 \%)$ \\
Emergence agitation & & \\
$3+4$ & $9(45.0 \%) *$ & $2(10.0 \%)$
\end{tabular}

Values are number of patients. Aono's four-point scale 3 or 4 are considered as emergence agitation. Group S: sevofluarane group, Group P: propofol group, ${ }^{*} \mathrm{P}<0.05$ compared with group $\mathrm{P}$.

\section{Results}

There were no statistically significant differences in the demographic and perioperative data of the 2 groups. NRS scores in the recovery room and the administered amount of fentanyl showed no statistically significant differences between the 2 groups (Table 2).

Emergence agitation occurred in 9 patients in group $\mathrm{S}$ (45.0\%), and 2 patients in group P $(10.0 \%)$, so the incidence of emergence agitation was higher in group $\mathrm{S}(\mathrm{P}=0.031)$ (Table 3$)$.

There were no differences observed in the incidence of severe emergence agitation, defined as an Aono's four-point scale score of 4 . There was no correlation between peak NRS score and peak Aono's four-point scale score, and there were no patients who displayed complications or side effects after the surgery in either group.

\section{Discussion}

Emergence agitation is a state of aggressive agitation that occurs temporarily in the process of emerging from anesthesia, 
and occurs most often during the early stages of emergence. The incidence of emergence agitation in adults is less frequent compared to pediatric patients, so little research has been done on adults. However, the occurrence of emergence agitation in adults results in a greater possibility of injury, and medical staff may not be able to restrain the agitation. Thus, there could may be the need for more medical staff available compared to pediatric patients, as problems can arise in the safety of both the patient and the medical staff. In the case of closed reduction of nasal bone fracture, in our study, there could be discomfort from the nasal packing performed to prevent post-operative bleeding.

The incidence of emergence agitation in adults differs according to the researcher. Lepouse et al. [5] reported it as 3\%, Yu et al. [7] as $21.3 \%$, Radtke et al. [8] as 5\%, and in our study it was $27.3 \%$. There are no clearly fixed criteria used to evaluate the occurrence of emergence agitation and its intensity in existing studies, but the Aono's four-point scale, Riker SedationAgitation Scale, Richmond Agitation-Sedation Scale, or personally categorized criteria have been used. The difference in incidence of emergence agitation according to researchers is considered to be from differences in criteria or differences in the standards used to define emergence agitation. There were no differences in incidence of emergence agitation between IV anesthetics and inhalation anesthetics according to Radtke et al.; however, in the research of Lepouse et al. and Yu et al., the incidence of emergence agitation was lower in the group that received IV anesthetics compared to the group that received sevoflurane. In our study, the propofol group had a lower incidence of emergence agitation compared to the sevoflurane group.

Sevoflurane has low blood-gas solubility, so fast inducement of and recovery from anesthesia is possible. In addition, the smell is pleasant, the drug causes less irritation to the airway than other inhalation anesthetics, and it has high cardiovascular stability; hence, it is a widely used drug. However, there are reports that sevoflurane has a higher rate of emergence agitation occurrence compared to other anesthetics $[1,2]$. In our study, also, group $\mathrm{S}$ exhibited a higher incidence of emergence agitation. The causes of emergence agitation when using sevoflurane are known to be rapid emergence time [9], young age [6], short anesthesia time, exterior stimulation, and pain [10], and combined administration of medication [11]. However, in our study, there were no differences between the groups in emergence time, age, anesthesia time, and post-operative pain, so the emergence agitation of sevoflurane cannot be explained. Cohen et al. [12] suggested that the cause of emergence agitation in patients who had undergone anesthesia with sevoflurane was that the difference in recovery speed within the nervous system increases the sensitivity to stimulation from the surrounding environment, creating a state of functional dissociation. Another theory is that emergence agitation occurs from the changes and relationship of gamma-aminobutyric acid $(\mathrm{GABA})_{\mathrm{A}}$ receptors in the central nervous system, and in the model of Sachedev and Kruck, the mechanism of excitement is explained to result from decreased inhibitory signals from the globus pallidus interna and substantia nigra, and the inability to suppress thalamocortical neurons and brain stem neurons due to disorder in the nervous system [13].

Propofol is an IV anesthetic with rapid excretion through the kidneys, and it is a drug with a rapid recovery time from general anesthesia as the patient emerges when the blood concentration decreases to lower than $50 \%$. However, in contrast to sevoflurane, propofol does not have a high incidence of emergence agitation in pediatric patients [12]. This is considered to result from the smooth recovery [3], remaining sedative effect in the early stages of emergence, and the euphoria caused by the drug [14]. Compared to inhalation anesthetics, propofol has a decreased occurrence of nausea and vomiting, and characteristically has a lower occurrence of hangover. This is considered to be related to the reduction in occurrence of emergence agitation.

Kim et al. [15] compared emergence agitation in pediatric patients who were administered propofol or sevoflurane as anesthesia for tonsillectomy. The incidence of emergence agitation in the propofol group was reported to be $60 \%$, while the incidence of emergence agitation in the sevoflurane group was $83 \%$. When compared with our study, the overall incidence of emergence agitation is higher in children than adults, but our study also found a lower incidence of emergence agitation in the propofol group than in the sevoflurane group. In the research of Kim et al., the incidence of severe emergence agitation with aggression, with an Aono's four-point scale score of 4 , was $10 \%$ with propofol and $20 \%$ with sevoflurane. In our study, emergence agitation with an Aono's four point scale score of 4 was not observed in the propofol group and showed a $10 \%$ occurrence in the sevoflurane group, so it is considered that severe emergence agitation occurs more frequently in children.

Previous studies have suggested that post-operative pain can be a risk factor related to emergence agitation [7], but in our study there was no correlation between the patient's NRS score and Aono's four-point scale score, and no significant difference in the amount of fentanyl administered between the 2 groups. There is a report that the incidence of emergence agitation decreased in pediatric patients who had received sevoflurane when ketorolac $0.5 \mathrm{mg} / \mathrm{kg}$ or fentanyl $1 \mu \mathrm{g} / \mathrm{kg}$ was administered [16], but there are also numerous reports suggesting that there is no relationship between pain control and emergence agitation [16-19].

Previous studies have reported methods to prevent emergence agitation. Abu-shahwan [20] reported that in pediatric patients 
who underwent general anesthesia with sevoflurane for MRI, emergence agitation could be reduced with additional administration of propofol $1 \mathrm{mg} / \mathrm{kg}$, and that in the case of performing general anesthesia with sevoflurane for dental treatment, additional administration of ketamine $0.25 \mathrm{mg} /$ $\mathrm{kg}$ reduced emergence agitation [21]. Isik et al. [22] reported that when sevoflurane was used for MRI, IV administration of dexmedetomidine $1 \mu \mathrm{g} / \mathrm{kg}$ reduced the occurrence of emergence delirium. Other than these, there are reports that fentanyl, nalbuphine, midazolam, and lidocaine are effective in reducing emergence agitation [22-24]. In our study, nalbuphine $10 \mathrm{mg}$ was to be IV administered every minute when a state of emergence agitation continued for more than 3 minutes, but emergence agitation in both groups was measured to last for about 1 minute, so additional administration of medication was unnecessary. A total of 24 patients reported an NRS score of 3 or higher; in these cases, fentanyl was administered to the patients by IV.

A limitation of this study is that we did not measure the patient's degree of anxiety before surgery. Lepouse et al. [5] reported that preoperative anxiety was a risk factor for emergence agitation, and in the research of Kain et al. [25], preoperative anxiety was related to delirium or changes in behavior after surgery. However, in our study we did not measure the degree of preoperative anxiety in the subjects. Furthermore, as all of the patients in our study received no preoperative medication, we did not consider whether the degree of preoperative anxiety or preoperative administration of drugs, such as benzodiazepines, could be related to the occurrence of emergence agitation.

In conclusion, the incidence of emergence agitation in adult patients, who underwent closed reduction of nasal bone fracture, was lower in the group that underwent general anesthesia using propofol than in the group that received sevoflurane. There was no correlation between postoperative pain and severity of emergence agitation. However, there is the need for further research on patients undergoing other surgeries to confirm whether propofol can reduce the incidence of emergence agitation in adult patients.

\section{References}

1. Yasuda N, Lockhart SH, Eger EI 2nd, Weiskopf RB, Liu J, Laster M, et al. Comparison of kinetics of sevoflurane and isoflurane in humans. Anesth Analg 1991; 72: 316-24.

2. Beskow A, Westrin P. Sevoflurane causes more postoperative agitation in children than does halothane. Acta Anaesthesiol Scand 1999; 43: 536-41.

3. Lapin SL, Auden SM, Goldsmith LJ, Reynolds AM. Effects of sevoflurane anaesthesia on recovery in children: a comparison with halothane. Paediatr Anaesth 1999; 9: 299-304.

4. Uezono S, Goto T, Terui K, Ichinose F, Ishguro Y, Nakata Y, et al. Emergence agitation after sevoflurane versus propofol in pediatric patients. Anesth Analg 2000; 91: 563-6.

5. Lepouse C, Lautner CA, Liu L, Gomis P, Leon A. Emergence delirium in adults in the post-anaesthesia care unit. Br J Anaesth 2006; 96: 747-53.

6. Aono J, Ueda W, Mamiya K, Takimoto E, Manabe M. Greater incidence of delirium during recovery from sevoflurane anesthesia in preschool boys. Anesthesiology 1997; 87: 1298-300.

7. Yu D, Chai W, Sun X, Yao L. Emergence agitation in adults: risk factors in 2,000 patients. Can J Anaesth 2010; 57: 843-8.

8. Radtke FM, Franck M, Hagemann L, Seeling M, Wernecke KD, Spies CD. Risk factors for inadequate emergence after anesthesia: emergence delirium and hypoactive emergence. Minerva Anestesiol 2010; 76: 394-403.

9. Wells LT, Rasch DK. Emergence "delirium" after sevoflurane anesthesia: a paranoid delusion? Anesth Analg 1999; 88: 1308-10.

10. Lynch EP, Lazor MA, Gellis JE, Orav J, Goldman L, Marcantonio ER. The impact of postoperative pain on the development of postoperative delirium. Anesth Analg 1998; 86: 781-5.

11. Veyckemans F. Excitation and delirium during sevoflurane anesthesia in pediatric patients. Minerva Anestesiol 2002; 68: 402-5.

12. Cohen IT, Finkel JC, Hannallah RS, Hummer KA, Patel KM. Rapid emergence does not explain agitation following sevoflurane anaesthesia in infants and children: a comparison with propofol. Paediatr Anaesth 2003; 13: 63-7.

13. Lindenmayer JP. The pathophysiology of agitation. J Clin Psychiatry 2000; 61 Suppl 14: 5-10.

14. Nakayama S, Furukawa H, Yanai H. Propofol reduces the incidence of emergence agitation in preschool-aged children as well as in school-aged children: a comparison with sevoflurane. J Anesth 2007; 21: 19-23.

15. Kim JH, Lee YC, Lee JN, Park YC. A comparative study of recovery characteristics between propofol-remifentanil and sevofluranenitrous oxide anesthesia in children. Korean J Anesthesiol 2006; 51: 709-14

16. Weldon BC, Bell M, Craddock T. The effect of caudal analgesia on emergence agitation in children after sevoflurane versus halothane anesthesia. Anesth Analg 2004; 98: 321-6.

17. Moore JK, Moore EW, Elliott RA, St Leger AS, Payne K, Kerr J. Propofol and halothane versus sevoflurane in paediatric day-case surgery: induction and recovery characteristics. Br J Anaesth 2003; 90: 461-6.

18. Cravero J, Surgenor S, Whalen K. Emergence agitation in paediatric patients after sevoflurane anaesthesia and no surgery: a comparison with halothane. Paediatr Anaesth 2000; 10: 419-24.

19. Lerman J, Davis PJ, Welborn LG, Orr RJ, Rabb M, Carpenter R, et al. Induction, recovery, and safety characteristics of sevoflurane in children undergoing ambulatory surgery. A comparison with halothane. Anesthesiology 1996; 84: 1332-40.

20. Abu-Shahwan I. Effect of propofol on emergence behavior in children after sevoflurane general anesthesia. Paediatr Anaesth 2008; 18: 55-9.

21. Abu-Shahwan I, Chowdary K. Ketamine is effective in decreasing the incidence of emergence agitation in children undergoing dental 
repair under sevoflurane general anesthesia. Paediatr Anaesth 2007; 17: 846-50.

22. Isik B, Arslan M, Tunga AD, Kurtipek O. Dexmedetomidine decreases emergence agitation in pediatric patients after sevoflurane anesthesia without surgery. Paediatr Anaesth 2006; 16: 748-53.

23. Dalens BJ, Pinard AM, Letourneau DR, Albert NT, Truchon RJ. Prevention of emergence agitation after sevoflurane anesthesia for pediatric cerebral magnetic resonance imaging by small doses of ketamine or nalbuphine administered just before discontinuing anesthesia. Anesth Analg 2006; 102: 1056-61.

24. Cohen IT, Hannallah RS, Hummer KA. The incidence of emergence agitation associated with desflurane anesthesia in children is reduced by fentanyl. Anesth Analg 2001; 93: 88-91.

25. Kain ZN, Caldwell-Andrews AA, Maranets I, McClain B, Gaal D, Mayes LC, et al. Preoperative anxiety and emergence delirium and postoperative maladaptive behaviors. Anesth Analg 2004; 99: 164854. 